\title{
Uso de herramientas digitales como estrategia para el desarrollo habilidades de análisis y razonamiento en los estudiantes de TIC
}

\section{Use of digital tools as a strategy for the development of analysis and reasoning skills in ICT students}

\author{
MALDONADO-MARTÍNEZ, Abish Amparo $\dagger^{*}$, GALICIA-ESCALANTE, Alejandra, APOLINAR- \\ PEÑA, José Jesús y HERRERA-CRUZ, Joel
}

Universidad Tecnológica del Valle de Toluca

ID $1^{\text {er }}$ Autor: Abish Amparo, Maldonado-Martínez / ORC ID: 0000-0002-9897-8570, CVU CONACYT ID: 679656

ID $1^{\text {er }}$ Coautor: Alejandra, Galicia-Escalante / ORC ID: 0000-0003-0688-7522, CVU CONACYT ID: 401191

ID $2^{\text {do }}$ Coautor: José Jesús, Apolinar-Peña / ORC ID: 0000-0002-9132-6676, CVU CONACYT ID: 677110

ID $3^{\text {er }}$ Coautor: Joel, Herrera-Cruz, / ORC ID: 0000-0003-3237-769X, CVU CONACYT ID: 680821

DOI: $10.35429 /$ JITC.2019.7.3.13.18

Recibido 06 de Enero, 2019; Aceptado 30 de Marzo, 2019

\section{Resumen}

Las competencias genéricas que la carrera de Tecnologías de la Información y Comunicación requiere que el estudiante pueda tener las habilidades de análisis y razonamiento para ser capaces de integrar y administrar tecnologías de la información y comunicaciones, que contribuyan a la productividad y el logro de los objetivos estratégicos de las organizaciones. Por lo que a través de herramientas digitales en el proceso de enseñanza aprendizaje, el docente contribuirá a desarrollar esas habilidades. Se analizan y proponen aquellas que contribuirán al objetivo bajo el modelo ADDIE (quien representa una guía descriptiva para la construcción de herramientas de formación y apoyo gracias al desarrollo de sus cinco fases que proceden de las iniciales de cada una de ellas en inglés) como primera fase; para continuar con la creación de diferentes actividades y estrategias que se aplicarán con los estudiantes y entonces medir el impacto hacia el desarrollo de éstas. Las habilidades de análisis y razonamiento contribuyen al pensamiento analítico, siendo importante porque constituye una de las bases para el pensamiento crítico, además que ayuda a resolver problemas que se presentan en la vida cotidiana.

Herramienta, análisis, razonamiento

\begin{abstract}
The generic competences that the career of Information and Communication Technologies require that the student can have the analysis and reasoning skills to be able to integrate and manage information and communications technologies, which contribute to productivity and the achievement of objectives Strategic organizations. So, through digital tools in the teaching-learning process, the teacher will contribute to develop those skills. Those that will contribute to the objective under the ADDIE model are analyzed and proposed (which represents a descriptive guide for the construction of training and support tools thanks to the development of its five phases that come from the initials of each of them) as first phase; to continue with the creation of different activities and strategies that will be applied with the students and then measure the impact towards their development. The analysis and reasoning skills contribute to analytical thinking, being important because it constitutes one of the bases for critical thinking, as well as helping to solve problems that arise in everyday life.
\end{abstract}

Tool, Analysis, Reasoning

Citación: MALDONADO-MARTÍNEZ, Abish Amparo, GALICIA-ESCALANTE, Alejandra, APOLINAR-PEÑA, José Jesús y HERRERA-CRUZ, Joel. Uso de herramientas digitales como estrategia para el desarrollo habilidades de análisis y razonamiento en los estudiantes de TIC. Revista de Tecnologías de la Información y Comunicaciones. 2019. 3-7: 13-18

\footnotetext{
* Correspondencia del Autor: abish.maldonado@utvtol.edu.mx)

$\uparrow$ Investigador contribuyendo como primer autor.
} 


\section{Introducción}

Conforme la tecnología avanza las practicas docentes también deberían ir avanzado, el estudiante universitario del siglo XXI demanda diferentes estrategias de aprendizaje acordes a lo que está acostumbrado a manejar cotidianamente, como el uso de herramientas digitales y aplicaciones. Ante este escenario, es importante iniciar una forma diferente de llevar en aula estrategias que apoyen a desarrollar las habilidades de análisis y razonamiento auxiliándonos de herramientas digitales. Dentro de la búsqueda de herramientas digitales se encontraron diferentes propuestas que poseen su versión de paga y gratuita, ofreciendo alternativas que ayudan a cumplir el objetivo de aprendizaje de los programas educativos.

\section{Justificación}

Los estudiantes de las instituciones de educación superior (IES), requieren hoy en día tener un aprendizaje significativo, pertinente que les ayude a adquirir conocimientos y competencias que les permita adherirse al campo laboral. Sin duda las habilidades de análisis y razonamiento en todos los estudiantes son necesarias, pero en los estudiantes de la carrera de Tecnologías de la Información y Comunicación, son básicas. Ya que es indispensable la solución de problemas y la toma de decisiones.

"Aunque la mayoría de los educadores consideran la habilidad de solución de problemas como el resultado de aprendizaje más importante, existen pocas prescripciones de diseño instruccional disponibles para diseñar la instrucción de solución de problemas y atraer a los estudiantes". (Jonassen, 1997). Por lo que con este proyecto al conocer las herramientas digitales on-line ayudará a que los docentes las empleen y ayuden a desarrollar en los estudiantes estas habilidades.

\section{Problema}

El objetivo de la carrera de Tecnologías de la Información es formar a profesionales con una visión global en el ámbito de la tecnología; implicando que, durante sus años de estudios, adquirirán competencias con las que puedan desarrollar su capacidad analítica, pero también la creativa para desarrollar y aplicar soluciones a través de sistemas informáticos e innovaciones tecnológicas.
El estudiante universitario del siglo XXI se caracteriza por ser un nativo digital, muy diverso social y culturalmente, conectado y a la vez solitario, su generación es muy diferente a la de sus padres; por lo que la manera de aprender ha cambiado y el docente ha de tomar un nuevo rol y utilizar diferentes estrategias y herramientas para enseñar.

Entre esas herramientas sin lugar a duda, se encuentran las digitales, ya que van de la mano con la forma natural de aprendizaje de los estudiantes universitarios actualmente.

\section{Hipótesis}

Emplear herramientas digitales para crear estrategias para el desarrollo habilidades de análisis y razonamiento en los estudiantes de TIC.

\section{Objetivos}

\section{Objetivo General}

Emplear herramientas digitales para crear estrategias para el desarrollo de habilidades de análisis y razonamiento con contenidos de las asignaturas que integran el plan de estudios en las carreras Técnico Superior Universitario en Tecnologías de la Información área Desarrollo de Software Multiplataforma y área Infraestructura de Redes Digitales de la Universidad Tecnológica del Valle de Toluca durante el periodo septiembre 2019 - agosto 2020.

\section{Objetivos específicos}

- Conocer y analizar los beneficios y las dificultades del uso de herramientas digitales dentro del aula para tener un aprendizaje significativo.

- Buscar las herramientas digitales en línea viables para las asignaturas del programa educativo, describiendo su aplicación y forma de empleo.

- Establecer los componentes que contendrán las estrategias de las diferentes asignaturas.

- Seleccionar los temas de las asignaturas y las herramientas digitales que ayudaran a desarrollar las habilidades de análisis y desarrollo. 
- Estructurar el contenido de las asignaturas dentro de la herramienta digital.

Capacitar a los docentes y estudiantes en el uso y aplicación de las herramientas digitales seleccionadas.

Analizar los resultados de las diferentes herramientas digitales, así como el impacto que tuvo en el aprendizaje en la comunidad estudiantil.

\section{Marco Teórico}

\section{Pensamiento Computacional}

Existen muchas acepciones acerca de la definición de pensamiento computacional, pero en fue utilizado la primera vez por Jeannette Wing en el año 2006. En el 2010 Wing junto con Jan Cuny, de la Fundación Nacional de Ciencias, y Larry Snyder, de la Universidad de Washington (Wing, 2011), dieron a conocer el siguiente concepto:

"Pensamiento computacional es el proceso de pensamiento donde están involucradas la formulación de los problemas y sus soluciones, donde las soluciones están representadas en una forma que se pueden llevar a cabo con eficacia por un agente de procesamiento de información (Wings, 2011, p.1)". Bolívar (2019) hace un compendio (tabla 1) donde muestra las definiciones de varios autores, donde podemos ver la importancia que los estudiantes del área de Tecnologías de la Información puedan desarrollar procesos del pensamiento (habilidades de análisis y razonamiento).

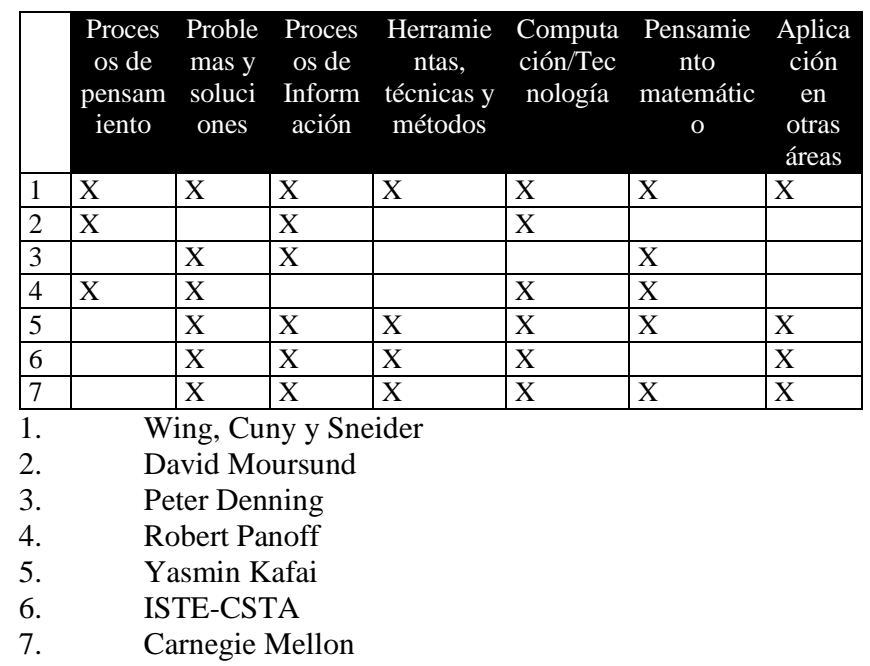

Tabla 1 Terminología relacionada con el pensamiento computacional

Fuente: Bolívar, 2019

\section{Habilidades de razonamiento y análisis}

El razonamiento y el análisis son procesos cognitivos básicos por medio del cual utilizamos y aplicamos nuestro conocimiento. Sin la posibilidad de hacer inferencias, el sistema de procesamiento humano se vería obligado a depender de un conocimiento específico y exacto para cada una de las situaciones con las que se encuentra. Las investigaciones sobre el razonamiento acuden a la lógica en busca de un criterio para evaluar el curso de estas inferencias y para identificar las leyes del conocimiento. (Saénz, Arrieta y Pardo, 2000).

Las habilidades de análisis son las diferentes capacidades que le permite a las personas construir y organizar sus conocimientos al momento de aplicarlos en las diferentes situaciones que se le presenten. por medio de esta habilidad podemos relacionar todo aquello que nos permite extraer nuestras propias conclusiones que nos van a hacer de mucha utilidad en el futuro. Es decir, nos ayudan a la toma de decisiones.

\section{Herramienta digital}

Son todos los recursos de software (algunos incluyen en su definición al hardware que contiene este software) presentes en computadoras y dispositivos relacionados, que permite realizar o facilitar todo tipo de actividades (Toledo y Hervás, 2009). En general las herramientas digitales permiten, dependiendo de cada caso:

Facilitar las comunicaciones a distancia entre personas. Por ejemplo: redes sociales, software de videoconferencias, etc.

Facilitar la educación, enriqueciéndola y haciéndola más entretenida. Por ejemplo: aplicaciones ofimáticas, enciclopedias en línea, creadores de presentaciones multimedia, etc.

Mejorar la organización de una empresa o institución.

Ser usadas en investigación.

- Completar bases de datos de cualquier tipo

Facilitar la realización de una tarea o actividad.

\section{Metodología a desarrollar}

MALDONADO-MARTÍNEZ, Abish Amparo, GALICIA-ESCALANTE, Alejandra, APOLINAR-PEÑA, José Jesús y HERRERA-CRUZ, Joel. Uso de herramientas digitales como estrategia para el desarrollo habilidades de análisis y razonamiento en los estudiantes de TIC. Revista de Tecnologías de la Información y Comunicaciones. 2019 


\section{Tipo de Investigación}

Investigación Aplicada

\section{Métodos Teóricos}

A continuación, se propone una metodología de análisis, diseño y desarrollo de ambientes educativos computarizados basados en Internet y aquellos elementos adicionales que deben tenerse en cuenta en estos modelos de instrucción.

La metodología consiste en un proceso iterativo de varias fases las cuales deben llevarse a cabo como lo determina la figura 2. Las cinco etapas que componen esta metodología son Análisis, Diseño, Desarrollo, Evaluación y Administración (ADDIE). Durante cada una de éstas, se plantean una serie de factores claves de éxito que expone Galvis (1998) en los procesos OLL\&T1 (aprendizaje y entrenamiento en línea), estos factores son críticos para el éxito del sistema y no se deben descuidar con el fin de evitar que éste fracase.

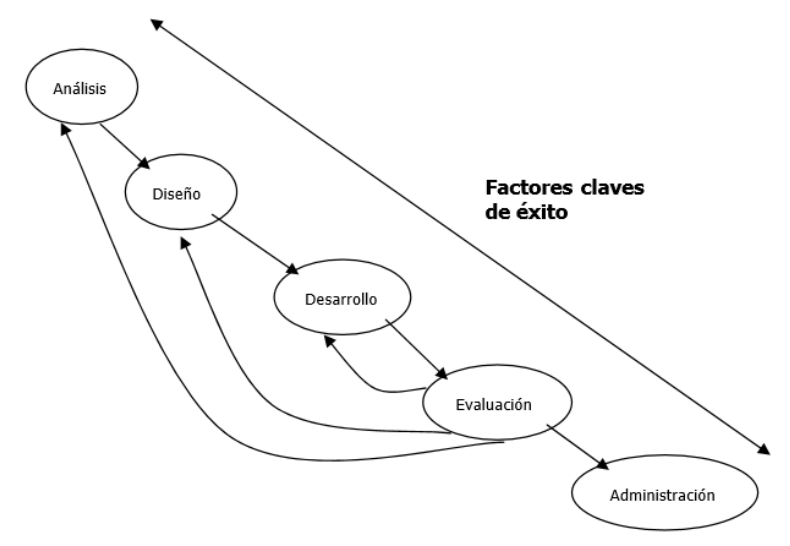

Figura 1 Metodología de análisis, diseño y desarrollo de ambientes educativos computarizados basados en Internet Fuente: Mendoza y Galvis, 1999

Las etapas se describirán a continuación:
A la par se analizarán las diferentes asignaturas y los temas de los planes de estudio que contribuyan al desarrollo de estas habilidades.

b) Diseño: El diseño de las estrategias de enseñanza - aprendizaje debe ser atractivo y de fácil manejo para los estudiantes, se busca que el diseño y las herramientas despierten el interés de su uso, facilitando el aprendizaje, los contenidos deben ser alineados a los programas de estudio.

c) Desarrollo: Inicialmente se dará capacitación a los docentes de las herramientas digitales utilizadas y brindar asesoría para que puedan emplearse en el aula.

d) Evaluación: Se desarrollará un instrumento de evaluación que permita detectar áreas de oportunidad en las estrategias empleadas, diseño, estructura, y el impacto de aprendizaje en los estudiantes

e) Administración: Se establecerá un programa permanente de asesoría a los docentes y se elaborará un portafolio de estrategias con las estrategias desarrolladas para su uso futuro.

\section{Metodología de Desarrollo}

Para conseguir el objetivo, se seguirán los pasos siguientes:

1. Inducir a los docentes en el uso de herramientas digitales como apoyo en su práctica enseñanza aprendizaje para lograr un aprendizaje significativo

2. Buscar y analizar el uso de herramientas digitales de acuerdo con los contenidos del programa educativo para lograr un aprendizaje significativo.

3. Seleccionar las herramientas digitales en línea viables para las asignaturas del programa educativo, describiendo su aplicación y forma de empleo.

4. Establecer los componentes que contendrán las estrategias de las diferentes asignaturas.

5. Seleccionar los temas de las asignaturas y las herramientas digitales que ayudaran a desarrollar las habilidades de análisis y desarrollo.

6. Estructurar el contenido de las asignaturas dentro de la herramienta digital. 
7. Capacitar a los estudiantes en el uso y aplicación de las herramientas digitales seleccionadas.

8. Analizar los resultados de las diferentes herramientas digitales, así como el impacto que tuvo en el aprendizaje en la carrera de Tecnologías de la Información área Desarrollo de Software Multiplataforma y área Infraestructura de Redes Digitales.

Cronograma de Actividades:

\begin{tabular}{|c|c|c|c|}
\hline \multirow[b]{2}{*}{ Actividades } & \multicolumn{3}{|c|}{ Periodos } \\
\hline & $\begin{array}{l}\text { Sep - } \\
\text { Dic } \\
2019\end{array}$ & $\begin{array}{l}\text { Ene - } \\
\text { Abr } \\
2020\end{array}$ & $\begin{array}{l}\text { May - } \\
\text { Ago } \\
2020\end{array}$ \\
\hline $\begin{array}{l}\text { Inducir a los docentes en } \\
\text { el uso de herramientas } \\
\text { digitales }\end{array}$ & & & \\
\hline $\begin{array}{l}\text { Buscar y analizar el uso } \\
\text { de herramientas digitales }\end{array}$ & & & \\
\hline $\begin{array}{l}\text { Seleccionar las } \\
\text { herramientas digitales en } \\
\text { línea }\end{array}$ & & & \\
\hline $\begin{array}{ll}\text { Establecer } & \text { los } \\
\text { componentes } & \end{array}$ & & & \\
\hline $\begin{array}{l}\text { Seleccionar los temas de } \\
\text { las asignaturas y las } \\
\text { herramientas digitales }\end{array}$ & & & \\
\hline Estructurar el contenido & & & \\
\hline $\begin{array}{lll}\begin{array}{l}\text { Capacitar } \\
\text { estudiantes }\end{array} & \text { a } & \text { los }\end{array}$ & & & \\
\hline $\begin{array}{lr}\text { Analizar los } & \text { resultados } \\
\text { de las } & \text { diferentes } \\
\text { herramientas } & \text { digitales } \\
\text { utilizadas } & \\
\end{array}$ & & & \\
\hline
\end{tabular}

\section{Resultados}

$\mathrm{Al}$ analizar las diferentes herramientas digitales, se encontró que las siguientes son algunas que ayudarán a cumplir el objetivo planteado ya que existen muchas herramientas digitales para ser empleadas en el aula. Ha ido creciendo en estos años una nueva clase de software cuyo objetivo es facilitar la creación, publicación y gestión de los materiales educativos en formato digital, que ha generado productos educativos muy importantes, pero que no desarrollan una adecuada función instructiva hacia la persona que las utiliza, y donde las diferencias están en su diseño y no en su uso.

Las herramientas propuestas son gratuitas y poseen su versión de paga, pero su versión gratuita ofrece muchas alternativas que ayudan a cumplir el objetivo.

\section{a) Mentimeter}

Sistema de creación de encuestas, que se pueden utilizar en las clases como, por ejemplo:

- $\quad$ Elección de la directiva del curso

- Votaciones para acuerdos

- Saber sí aprendieron un contenido

- Crear cuestionarios usando las diferentes plantillas que ofrece, con variadas preguntas, para utilizarlas en el aula y los alumnos "voten" la respuesta más adecuada en sus dispositivos móviles.

- Crear presentaciones muy divertidas e innovadoras, los estudiantes se mantendrán muy participativos $\mathrm{y}$, por lo tanto, mejorarán los resultados de aprendizaje,

Hacer que una evaluación formativa sea más fácil con resultados imparciales al permitir que los estudiantes respondan anónimamente.

\section{b) GoConqr}

Entorno de estudio personalizado online y gratuito que te ayuda a mejorar tu aprendizaje. GoConqr incluye herramientas de aprendizaje que permiten crear, compartir y descubrir:

$\begin{array}{ll}- & \text { Mapas Mentales } \\ - & \text { Fichas de Estudio } \\ - & \text { Apuntes Online } \\ - & \text { Tests }\end{array}$

c) Educaplay

Es una plataforma para la creación de actividades educativas multimedia, caracterizadas por sus resultados atractivos y profesionales. Permitiendo realizar:

$\begin{array}{ll}- & \text { Crucigrama } \\ - & \text { Dictados } \\ - & \text { Completar } \\ - & \text { Diálogo } \\ - & \text { Mapas interactivos } \\ - & \text { Adivinanzas } \\ - & \text { Relacionar } \\ - & \text { Test } \\ - & \text { Sopa de letras } \\ - & \text { Ordenar palabras } \\ - & \text { Videoquiz }\end{array}$

MALDONADO-MARTÍNEZ, Abish Amparo, GALICIA-ESCALANTE Alejandra, APOLINAR-PEÑA, José Jesús y HERRERA-CRUZ, Joel. Uso de herramientas digitales como estrategia para el desarrollo habilidades de análisis y razonamiento en los estudiantes de TIC. Revista de Tecnologías de la Información y Comunicaciones. 2019 


\section{d) Flipgrid}

Es una plataforma de aprendizaje social que permite a los profesores y estudiantes interactuar utilizando el vídeo. Básicamente, permite elaborar cuestionarios, pero da un paso más, pues nos sirve para grabar las opiniones y respuestas de los alumnos:

Desarrolla la capacidad de expresión de los estudiantes.

Puede ayudar a la evaluación de una actividad.

Se puede pedir su opinión sobre una lectura, una película que hayas visualizado, un tema tratado en clase.

Se empoderas al alumnado dándole voz y voto.

Aumenta la confianza de los alumnos.

\section{Agradecimiento}

El trabajo fue apoyado por la Dirección de Tecnologías de la Información y Comunicación y Secretaría Académica de la Universidad Tecnológica del Valle de Toluca.

\section{Conclusiones}

Los roles de los estudiantes y docentes del siglo XXI han cambiado, hoy en día ante nativos digitales los procesos de enseñanza aprendizaje deben cambiar y ayudar a potencializar las habilidades de razonamiento y análisis en los estudiantes de la carrera de TIC, por lo que deben emplearse herramientas digitales para estar a tono con la manera en que aprenden los estudiantes.

Una vez que se analizaron las herramientas digitales, es necesario capacitar a los docentes y elaborar las estrategias a seguir.

Una vez aplicadas en el aula mediremos el impacto que hayan tenido en el aprendizaje de los estudiantes de las carreras Técnico Superior Universitario en Tecnologías de la Información área Desarrollo de Software Multiplataforma y área Infraestructura de Redes Digitales de la Universidad Tecnológica del Valle de Toluca durante el periodo septiembre 2019 - agosto 2020.

\section{Referencias}

Bolívar, M. I. S. (2019). Experiencias y estrategias educativas con TIC para el desarrollo del pensamiento computacional en Iberoamérica. Pensamiento Actual, 19(32), 1227.

Jonassen, D. H. (1997). "Instructional Design Models for Well-Structured and Ill-Structured Problem-Solving Learning Outcomes", Educational Technology Research and Development, Vol. 45, No. 1, 1997.

Mendoza, P. y Galvis, A. (1999). Disponible en http://www.revistavirtualpro.com/biblioteca/am bientes-virtuales-de-aprendizaje-unametodologia-para-su-creacion. Consultado 15 de agosto de 2019

Salinas, J. y Urbina, S. (2007). Bases para el diseño, la producción y la evaluación de procesos de Enseñanza-Aprendizaje mediante nuevas tecnologías. En J. Cabero (coord.) Nuevas Tecnologías aplicadas a la Educación (pp. 41-62). Madrid: McGraw Hill.

Sáenz, I., Arrieta M. \& Pardo E. (2000). Por los Caminos de la Lógica: Lógica y Conjuntos en E.G.B. Madrid: Síntesis

Toledo, P. y Hervás, C. (Coord.) (2009). El software libre en los contextos educativos. Sevilla: Eduforma

UNESCO (2013). Directrices para las políticas de aprendizaje móvil. Organización de las Naciones Unidas para la Educación, la Ciencia y la Cultura. París: Francia.

Wing, J. M. (2011). Computational Thinking: What and Why? Consultado: septiembre 9 de 2019, disponible en: http://www.cs.cmu.edu/ CompThink/resources/ TheLinkWing.pdf 